\title{
The effect of elastic support location on natural frequency of pipe conveying fluid
}

\author{
Zhou Ping*, and Li Yan Qing \\ China Ship Scientific Research Center \\ China \\ zhouping0830@163.com
}

\author{
Zhang Zi Jun \\ China Academy of Launch Vehicle Technology \\ China
}

\begin{abstract}
The governing equation of pipe conveying fluid was deduced based on Timoshenko beam model. Wave method was applied to solve the governing equation. In the process, wave reflection matrices were obtained for three typical boundary conditions and the wave scattering model was established for the intermediate elastic support. Based on the above model, the wave characteristic equation for pipe conveying fluid with intermediate elastic support was obtained through wave train closure principle. In the example, the natural frequencies and critical fluid velocities were calculated for pipe conveying fluid with the intermediate elastic support at different locations.
\end{abstract}

Keywords-pipe conveying fluid, natural frequency, wave propagation method, timoshenko beam

\section{INTRODUCTION}

Fluid-conveying pipe system is widely used in engineering. Destruction of pipe caused by fluid-induced vibration is quite common. These years, many researchers focused on the fluid structure interaction and studied the vibration of pipe conveying fluid and gained a lot of outstanding achievements in linear and non-linear fields. On the foundation of these researches, Wadham-Gagnon et al researched the dynamics of a cantilevered pipe with an end mass through nonlinear motion equation [1]. Ghayesh et al studied the nonlinear dynamics of cantilevered extensible pipes conveying fluid [2]. Huang et al researched the natural frequency of fluid conveying pipe with different boundary conditions, and obtained the different form functions of the pipe with different boundary conditions [3]. On the other hand, researchers have proposed various calculating methods for the existed vibration models. Lu and Lee studied the dynamic instability of a long-pipe system and developed a more direct method to classify the stability conditions and ranges [4]. Pramila et al studied the dynamic stability of a short pipe using the finite element method [5]. In the research, the pipe element was simulated by Timoshenko beam element. Tan and Kang have used the wave method in the vibration research of a rotating Timoshenko shaft system [6]. Li et al used the wave method to research the natural frequency of multi-span pipe conveying fluid [7].

In practice, many pipe systems have intermediate elastic supports. This paper studied the free vibration of pipe conveying fluid with intermediate elastic support through wave method.

Project support: National key R \& D plan (2016YFc0300603) State Key Laboratry of Deep-sea Manned Vehicles
The Timoshenko beam model is used here to deduce the motion equation of the pipe, which is based on the research of Laithier and Paidoussis [8]. The influences of support location on the natural frequency and the critical fluid velocity of the pipe were discussed. Contrasts are made to validate the wave method. The results can provide a reference for the fluid-induced vibration of pipes with elastic intermediate supports. And the method used in this paper provides a new thought for the dynamic research of multi-span pipes.

\section{MOTION EQUATION}

Neglect the internal damping and external load, the lateral vibration equation of pipe conveying fluid can be written as the following form [8].

$$
\frac{\partial Q}{\partial x}-\left(m_{f}+m_{p}\right) \frac{\partial^{2} w}{\partial t^{2}}-m_{f} V^{2} \frac{\partial^{2} w}{\partial x^{2}}-2 m_{f} V \frac{\partial^{2} w}{\partial x \partial t}=0
$$

For a long pipe, the deformation of cross section can be neglected, so the pipe can be modeled as a beam. In order to consider the rotating inertia of pipe section, we use the Timoshenko beam to model pipe. The motion equations for Timoshenko beam are,

$$
\left\{\begin{array}{l}
M=E I \frac{\partial \varphi}{\partial x} \\
Q=k_{0} A G\left(\frac{\partial w}{\partial x}-\varphi\right) \\
\frac{\partial M}{\partial x}+Q=\rho I \frac{\partial^{2} \varphi}{\partial t^{2}}
\end{array}\right.
$$

For a thin walled pipe $k_{0}=2(1+\mu) /(4+3 \mu)$. Set that,

$$
\varphi=\varphi_{0} e^{i(-k x+\omega t)}
$$

where, $\varphi_{0}$ is the undetermined coefficient, $k$ is wave number, $\omega$ is circular frequency, and $i=\sqrt{-1}$. the relation between wave number and frequency can be obtained. 


$$
\begin{aligned}
& E I\left(k_{0} A G-m_{f} V^{2}\right) k^{4}+2 m_{f} V E I \omega k^{3} \\
& -\left\{\left[\left(k_{0} A G-m_{f} V^{2}\right) \rho I+m E I\right] \omega^{2}+k_{0} A G m_{f} V^{2}\right\} k^{2} \\
& +2 m_{f} V\left(k_{0} A G-\rho I \omega^{2}\right) \omega k+m \omega^{2}\left(\rho I \omega^{2}-k_{0} A G\right)=0
\end{aligned}
$$

Equation (4) is the so-called dispersion equation. Obviously, there are four roots of (4) with respect to $k$. These roots denote different wave motion. Set $k_{1}, k_{2}$ denote left propagated wave numbers and $k_{3}, k_{4}$ denote right propagated wave numbers.

Substitute wave number $k$ into (3), then the following result can be obtained.

$$
\varphi=\sum_{j=1}^{4} \varphi_{j} e^{i\left(-k_{j} x+\omega t\right)}
$$

Then the wave form of transverse displacement $w$, shear force $Q$ and bending moment $M$ can be expressed as,

$$
\left\{\begin{array}{l}
w=\sum_{j=1}^{4} \frac{\rho I \omega^{2}-E I k_{j}^{2}-k_{0} A G}{i k_{j} k_{0} A G} \varphi_{j} e^{i\left(-k_{j} x+\omega t\right)} \\
Q=\sum_{j=1}^{4}\left(E I k_{j}^{2}-\rho I \omega^{2}\right) \varphi_{j} e^{i\left(-k_{j} x+\omega t\right)} \\
M=\sum_{j=1}^{4}-E I i k_{j} \varphi_{j} e^{i\left(-k_{j} x+\omega t\right)}
\end{array}\right.
$$

Equation (5) and (6) both have the term $e^{i \omega t}$. For compact purpose, it will be suppressed in following parts.

\section{WAVE METHOD}

From the view of wave theory, when the elastic wave propagates along the pipe, reflection and transmission will occur at the discontinuity of material. At the boundary of the pipe, due to no other medium, the wave will be reflected only. Wave propagation matrix, reflection matrix and transition matrix are used to denote the above physical processes.

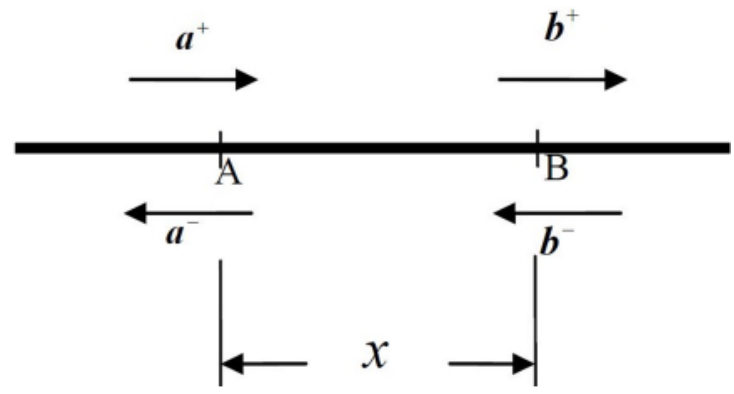

Fig. 1. Wave propagation sketch.

As shown in Fig. 1, the wave motions at point $\mathrm{A}$ are represented by $\boldsymbol{a}^{+}$and $\boldsymbol{a}^{-}$. In the same way, $\boldsymbol{b}^{+}$and $\boldsymbol{b}^{-}$denote the wave motions at point B. superscript ' + ' and '-' denote the right and left going waves respectively.

$$
b^{+}=\left[T_{r}\right] a^{+}, \quad a^{-}=\left[T_{l}\right] b^{-}
$$

where,

$$
a^{+}=\left\{\begin{array}{l}
a_{3} \\
a_{4}
\end{array}\right\}, \quad a^{-}=\left\{\begin{array}{l}
a_{1} \\
a_{2}
\end{array}\right\}, b^{+}=\left\{\begin{array}{l}
b_{3} \\
b_{4}
\end{array}\right\}, \quad b^{-}=\left\{\begin{array}{l}
b_{1} \\
b_{2}
\end{array}\right\}
$$

In (7), $\left[T_{r}\right]$ and $\left[T_{l}\right]$ denote propagation matrices of right and left going wave, respectively. Due to the Coriolis force, the traveling velocities of the left- and right-traveling waves are different. So the propagation matrices in these two directions are different too, i.e. $\left[T_{r}\right] \neq\left[T_{l}\right]$. This result is different with that in the research of Tan and Kang [6] which contains only one medium. Fig. 2 shows the wave reflection at the simply supported end. $\varphi^{+}, \varphi^{-}$denote the incident and reflected waves respectively. $[R]$ denotes the reflection matrix.

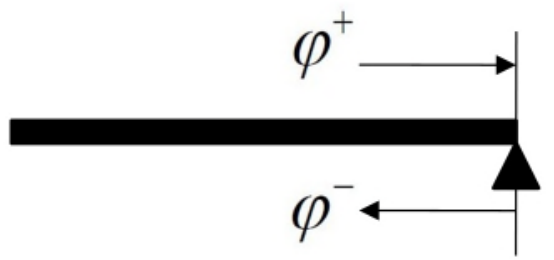

Fig. 2. Wave reflection at a simply supported end.

The relation between the incident and reflected wave can be expressed as follows.

$$
\varphi^{-}=[R] \varphi^{+}
$$

Here, take a simply supported pipe with intermediate elastic support as an example to deduce the wave characteristic equation for pipe conveying fluid. As shown in Fig. 3, the intermediate support divides the pipe into two sections. Using $\left[T_{1}\right]$ and $\left[T_{2}\right]$ represent the propagation matrices of the left and right sections, respectively.

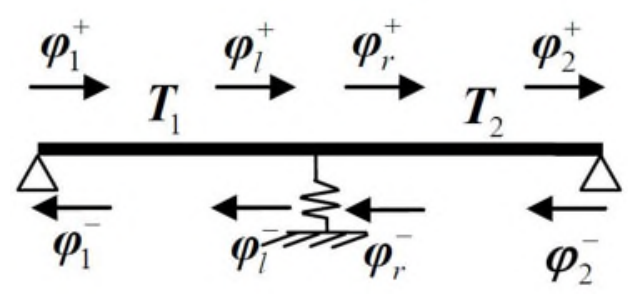

Fig. 3. Wave motion in pipe with intermediate support.

The wave characteristic equation with respect to $\varphi_{1}^{+}$for the whole pipe as follows.

$$
\left(\left[R_{1}\right]\left[T_{1 l}\right]\left[R_{l}\right]\left[T_{1 r}\right]-[I]\right) \varphi_{1}^{+}=0
$$


Here, $\boldsymbol{I}$ is a unit matrix. If (10) has non-trivial solution, the following determinant must equal to zero.

$$
h(\omega)=\operatorname{det}\left(\left[R_{1}\right]\left[T_{1 l}\right]\left[R_{l}\right]\left[T_{1 r}\right]-[I]\right)=0
$$

The natural frequencies can be calculated from (11).

\section{CALCULATION AND DISCUSSION}

\section{A. Natural Frequency}

As shown in Fig. 4, the simply supported pipe has an intermediate elastic support. In the example, we calculate the natural frequencies of pipe with the intermediate support at different locations when the fluid velocity is $10 \mathrm{~m} / \mathrm{s}$. The results are listed in Table I.

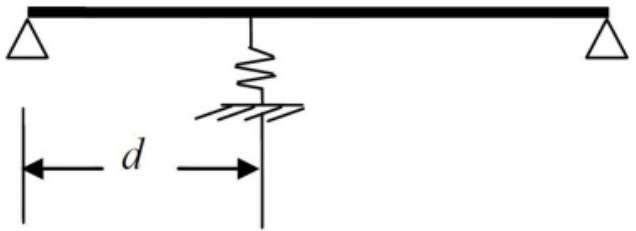

Fig. .4 Simply supported pipe with intermediate support.

TABLE 1. NATURAL FREQUENCIES OF PIPE UNDER DIFFERENT INTERMEDIATE SUPPORT LOCATION (RAD/S).

\begin{tabular}{llll}
\hline$d$ & $\omega_{1}$ & $\omega_{2}$ & $\omega_{3}$ \\
\hline 1 & 2.032 & 6.626514 & 13.88097 \\
1.5 & 2.278 & 7.494431 & 15.7502 \\
2 & 2.584 & 8.545455 & 17.34234 \\
2.5 & 2.968 & 9.610156 & 14.23669 \\
3 & 3.448 & 9.088452 & 13.1982 \\
3.5 & 3.998 & 7.440622 & 15.24161 \\
4 & 4.334 & 6.700246 & 17.3407 \\
\hline
\end{tabular}

We can conclude that when the intermediate support located at the midpoint of the simply supported pipe, it has a highest $1 \mathrm{st}$ order natural frequency. But this is not valid any more to the 2 nd or other higher orders. This conclusion can also be obtained through considering the mode shapes of the simply supported pipe. To the 1st order mode shape, when the intermediate support located at the midpoint of the pipe, it restrains the largest deformation point of the pipe (to a simply supported pipe, the largest deformation occur at the midpoint). So the mode strength reaches a highest value. But to the higher order mode shapes, this does not work anymore.

\section{B. Critical Fluid Velocity}

When the fluid velocity increases, the natural frequency will decrease. In other words, the flowing fluid can weaken the pipe stiffness. This has been validated by Paidoussis [9]. Through calculation, the critical velocities related to buckling of the pipe with different intermediate elastic supports can be obtained, as shown in Fig. 5.

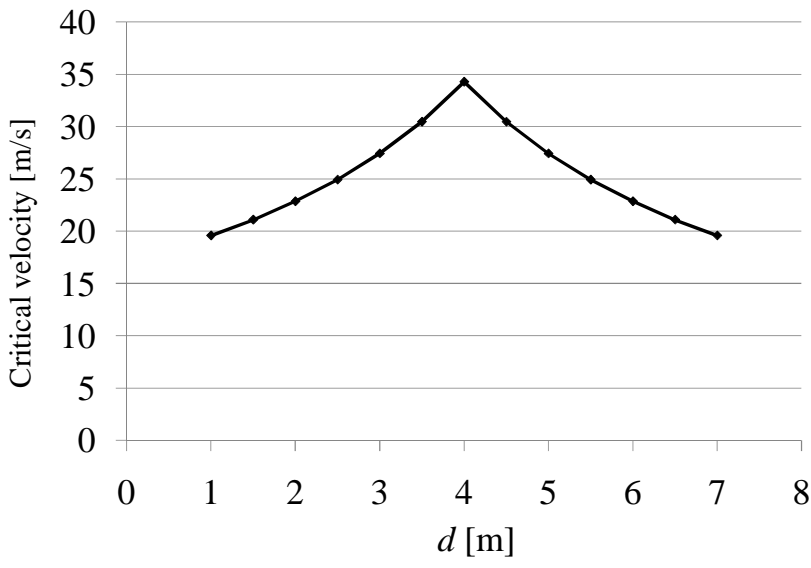

Fig. 5. Curve of critical velocity versus intermediate support position.

Fig. 5 shows a symmetrically inversely proportional relation between $\mathrm{d}$ and the critical velocity $V_{c r}$, i.e.

$$
V_{c r} \propto \frac{1}{\max (d, L-d)}
$$

\section{CONCLUSION}

The wave propagation method was applied to analyze the fluid structure interaction and ensuing vibration of pipe with intermediate elastic support. The wave reflection and scattering model was established at pipe boundaries and intermediate support, respectively. Based on above models, the wave characteristic equation of pipe conveying fluid was obtained. In the example, natural frequencies with different locations of intermediate elastic support of pipe were calculated. The results show that the highest critical fluid velocity appears when the intermediate support moves to the midpoint of pipe. The results in this paper agree well with the research of Paidoussis [9]. So the wave propagation method is valid. This provides a reference to the research of the multi-span pipe.

\section{REFERENCES}

[1] A. Wadham-Gagnon, M. P. Paidoussis, C. Semler, "Dynamics of Cantilevered Pipes Conveying Fluid. Part 1: Nonlinear Equations of Three-Dimensional Motion”, J. Fluid. Struct. vol. 23, no. 4, pp. 545-567, 2007.

[2] M.H. Ghayesh, M.P. Paidoussis, and M. Amabili, "Nonlinear Dynamics of Cantilevered Extensible Pipes Conveying Fluid", J. Sound Vib. vol. 332, no. 24, pp. 6405-6418, 2013.

[3] Y.M. Huang, Y.S. Liu, B.H. Li, Y.J. Li, and Z.F. Yue, "Natural Frequency Analysis of Fluid Conveying Pipeline with Different Boundary Conditions", Nucl. Eng. Des. vol. 240, no. 3, pp. 461-467, 2010.

[4] P. Lu, H.P. and Lee, "A Treatment for the Study of Dynamic Instabilities of Fluid-Conveying Pipes", Mech. Res. Commun. vol. 36, no. 6, pp. 742-746, 2009.

[5] A. Pramila, J. Laukkanen, and S. Liukkonen, "Dynamics and Stability of Short Fluid-Conveying Timoshenko Element Pipes", J. Sound Vib. vol. 144, no. 3, pp. 421-425, 1991.

[6] C.A. Tan, and B. Kang, "Free Vibration of Axially Loaded, Rotating Timoshenko Shaft Systems by the Wave-Train Closure Principle", Int. J. Sol. Struct. vol. 36, no. 26, pp. 4031-4049, 1999 
[7] B.H. Li, H.S. Gao, H.B. Zhai, Y.S. Liu, and Z.F. Yue, "Free Vibration Analysis of Multi-Span Pipe Conveying Fluid with Dynamic Stiffness Method”, Nucl. Eng. Des. vol. 241, no. 3, pp. 666-671, 2011.

[8] B.E. Laithier, and M.P. Paidoussis, "The Equations of Motion of Initially Stressed Timoshenko Tubular Beams Conveying Fluid", J. Sound Vib. vol. 79, no. 2, pp. 175-195, 1981.

[9] M.P. Paidoussis, Fluid-Structure Interactions: Slender Structures and Axial Flow, Academic Press, London, 1998. 